\title{
Gradhiva
}

GRADHIV

Revue d'anthropologie et d'histoire des arts

$2 \mid 2005$

Autour de Lucien Sebag

\section{L'Aigle et le Corbeau}

Quand les Aborigènes s'invitent à la table des anthropologues...

Eaglehawk and Crow. When Aborigines confront to the Anthropologists table

\section{Bastien Bosa}

\section{OpenEdition}

Journals

Édition électronique

URL : http://journals.openedition.org/gradhiva/463

DOI : $10.4000 /$ gradhiva.463

ISSN : $1760-849 X$

Éditeur

Musée du quai Branly Jacques Chirac

Édition imprimée

Date de publication : 1 novembre 2005

Pagination : $31-47$

ISBN : 2-915-133-10-7

ISSN : 0764-8928

Référence électronique

Bastien Bosa, «L'Aigle et le Corbeau », Gradhiva [En ligne], 2 | 2005, mis en ligne le 10 décembre 2008, consulté le 19 avril 2019. URL : http://journals.openedition.org/gradhiva/463 ; DOI : 10.4000/ gradhiva.463

Ce document a été généré automatiquement le 19 avril 2019.

(c) musée du quai Branly 


\section{L'Aigle et le Corbeau}

Quand les Aborigènes s'invitent à la table des anthropologues...

Eaglehawk and Crow. When Aborigines confront to the Anthropologists table

\section{Bastien Bosa}

We should move beyond treating modernity, liberalism, citizenship, or bourgeois equality as if

they were fixed and self-contained doctrines unaffected by the appropriations and reformulations given to them by processes of political mobilisation in Asia, Africa or Europe itself.

Frederick Cooper, « Conflict and connection : rethinking colonial African history », 1994. A-t-on quitté ce monde factice d'idées prises en l'air, à part, comme se suffisant à elles-mêmes, pour replacer cette fonction intellectuelle dans l'ensemble de vie humaine et sociale où seulement elle peut être comprise avec réalité et soumise aux interdépendances essentielles?

François Simiand, « À propos de l'Histoire des idées », 1903.

«Vous savez sans doute que la conférence de l'Institut Australien des études Aborigènes se tient à Canberra en mai. Peut-être avez-vous l'intention d'y participer, voire d'y présenter une contribution.

Pourquoi cette conférence a-t-elle lieu ? Quel impact [signifiance] aura-t-elle sur la compréhension des sociétés humaines? Quel impact sur les Aborigènes et sur notre position dans le monde? Quel impact sur les événements qui traversent aujourd'hui la société australienne?

Nous pensons que cette conférence n'a de contribution positive à offrir ni à la société australienne en général, ni à la position des Aborigènes en particulier. »

1 Tels étaient les premiers mots d'une lettre de cinq pages ${ }^{1}$ adressée en mars 1974 à l'ensemble des participants potentiels à l'assemblée bisannuelle de l'Institut Australien 
des Études Aborigènes (AIAS, voir encadré) qui devait se tenir quelques mois plus tard. Cette lettre, rédigée par un groupe intitulé The Eaglehawk and Crow, portait la signature de six personnes: Terry Widders, Peter Thomson, Gary Williams, Lyn Thomson, Bob Bellear et Len Watson. Tous, à l'exception de Peter Thomson'², étaient aborigènes et avaient participé, d'une façon ou d'une autre, au mouvement politique de contestation aborigène qui a traversé l'Australie entre 1969 et $1974^{3}$. Et c'est bien à ce titre qu'ils s'exprimaient :

« À l'exception d'une personne, nous sommes tous aborigènes et nous ne pouvons pas accepter que les Aborigènes soient confinés dans des rôles de sujets passifs, pour des études douteuses et qui pourtant sont faites à grands frais quand les besoins de la population restent énormes. "

Le groupe s'était donné pour tâche de jeter un regard critique sur les actions des autorités par rapport aux Aborigènes. Ses membres se livrèrent notamment à une critique de la politique de déplacement [relocation] des populations aborigènes, qu'ils percevaient comme une continuation forcée de la politique d'assimilation ${ }^{4}$, et certains prirent part à une enquête sur la perte de terres aborigènes liée au démantèlement des réserves ${ }^{5}$. Le groupe était néanmoins très informel. Comme me l'a expliqué Peter Thomson au cours de notre entretien :

«L'idée n'a jamais été de faire un comité... C'était simplement un petit groupe qui utilisait l'idée de "conflit créatif" pour apporter sa contribution à quelques débats de l'époque $»^{6}$.

Le nom du groupe lui-même - L'Aigle et le Corbeau - est intéressant. Deux explications concurrentes m'en ont été données. Peter Thomson m'a expliqué qu'il avait été choisi pour évoquer le «conflit créatif » entre les deux oiseaux du "temps du rêve ${ }^{7}$ ». Gary Williams se gardait, lui, de toute référence au « temps du rêve » et soulignait la volonté du groupe, très pragmatique, d'associer dans ses investigations les qualités de l'aigle, hauteur et vision globale des choses, à celles du corbeau, dont le vol à ras de terre permet de saisir les aspérités du terrain. 
De l'assimilation au « Black Power » : Gary Williams sur la couverture de Dawn, le journal de propagande de l'Aboriginal Welfare Board en mai 1963, comme symbole de la réussite de la politique d'assimilation.

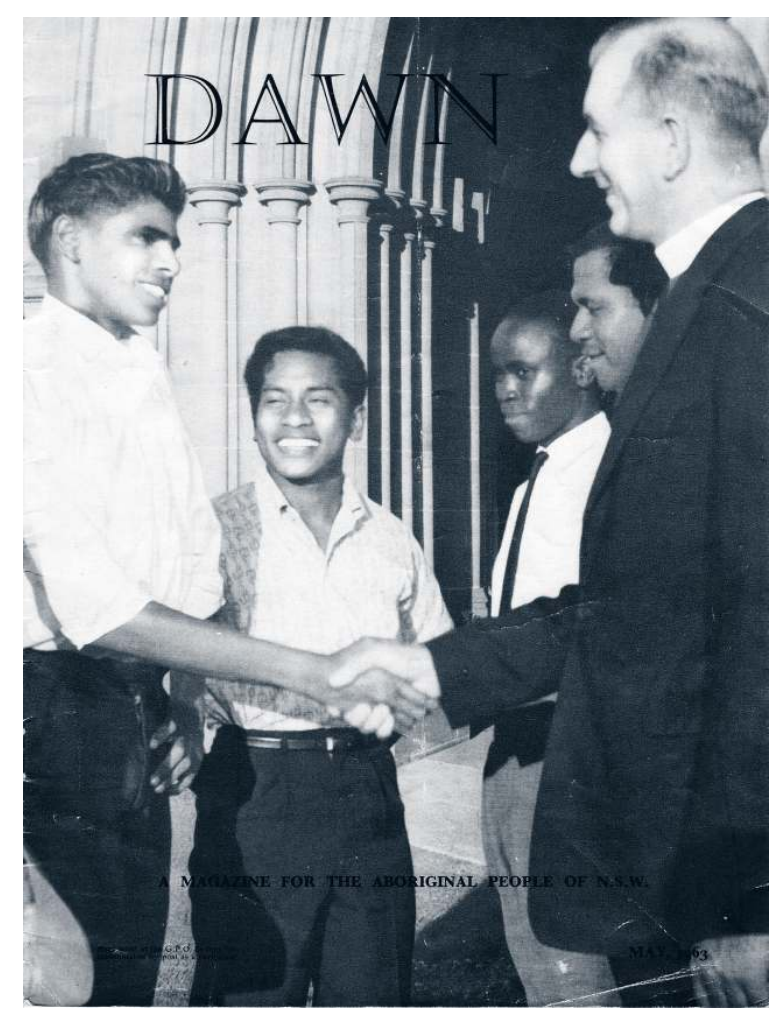

(c) Bob Gould, The Catholic Weekly, Sydney, Department of Aboriginal Affairs, Australian Institute of Aboriginal and Torres Islander Studies. DR.

4 Cette lettre, accompagnée des réponses qu'elle a suscitées, permet un questionnement renouvelé sur le problème des rapports entre science et politique. Alors que la question est généralement abordée sous l'angle des usages politiques de la science (Gaïti 2002), nous essaierons de montrer que cette lettre relève moins d'une sollicitation militante de la science (l'appropriation et l'usage de ressources scientifiques, souvent instrumentalisées et transformées, à l'intérieur du monde politique) que d'une interpellation des anthropologues par un groupe de militants (la contestation politique du monde scientifique).

5 La lettre constitue un document d'autant plus remarquable que les écrits sont rares où le rôle de l'anthropologie est mis en question par ceux qui en sont les " objets ${ }^{8}$ ». Le point de vue des " colonisés ", lorsqu'il apparaît, est généralement vu à travers le prisme des écrits des seuls «Blancs ». La lettre de l'Eaglehawk and Crow donne accès au contraire à un point de vue aborigène non médiatisé. Ce qui ne veut évidemment pas dire qu'elle représente "le regard aborigène authentique» sur l'anthropologie. D'une part, les rédacteurs appartenaient à une fraction bien particulière de la population aborigène, et c'est le regard particulier de ce groupe que la lettre nous permet de mettre en perspective. D'autre part, il est évident que la présence de Peter Thomson et sa connaissance du fonctionnement des « études aborigènes » ont eu un impact tout à fait décisif9.

6 Nous commencerons par présenter la lettre elle-même dans ses grandes lignes, puis nous détaillerons les nombreuses réponses qu'elle a suscitées. La lettre permet en effet de 
saisir, en miroir, certaines des tensions traversant le champ anthropologique australien à l'époque.

7 Nous réfléchirons ensuite aux motivations des militants, en essayant de prendre en compte à la fois leur trajectoire et leur position dans le champ politique australien. Nous interrogerons en conclusion les effets d'une telle interpellation, indépendamment des motivations de ses auteurs, sur la pratique anthropologique elle-même.

Qu'est-ce que l'AIAS?

L'Institut Australien des Études Aborigènes fut formellement établi en $1964^{10}$ afin de combler les lacunes du savoir produit en Australie sur les Aborigènes. Il fut mis en place dans une logique "d'anthropologie de sauvetage » (ce que les anglophones appellent rescue ou salvage anthropology), excluant explicitement du champ de ses investigations l'étude du "changement social», ainsi que les problèmes de politiques publiques. L'objet de l'AIAS tel que conçu par son instigateur, William Wentworth, député conservateur, était d'enregistrer ce qui avait subsisté de la culture aborigène traditionnelle, avant que tout ne disparaisse. Cet objectif fut repris à son compte par Fred McCarthy, premier directeur de l'Institut. Quelques changements se produisirent au début de la décennie 1970, notamment du fait du remplacement de McCarthy par Peter Ucko à la direction de l'AIAS en 1971. Symbole de cette modernisation, la conférence de 1974 devait être un événement international de trois semaines, par opposition à l'habituel séminaire régional de deux jours.

\section{La lettre}

Trois grandes critiques peuvent être dégagées.

\section{L'enfermement dans le monde universitaire}

9 Un premier ensemble de critiques formulées dans la lettre concernait le fonctionnement du monde académique. Les signataires mettaient en cause le hiatus entre, d'un côté, les anthropologues et, de l'autre, la société en général et le groupe de leurs enquêtés en particulier. Ils accusaient les anthropologues de se soucier davantage de leur propre carrière que de l'intérêt des populations qu'ils étudiaient. Le jugement était sévère :

« $\mathrm{Au}$ mieux, la conférence permettra à des universitaires de se retrouver et d'échanger des faits ésotériques ou des théories abstraites à propos de leurs "fascinants objets d'études". Ils réussiront peut-être même à dégager de nouvelles abstractions sur les sociétés humaines, ce qui renforcera leur prétention [assumed importance] ainsi que leur foi dans la pertinence de leur recherche.

$\mathrm{Au}$ pire, cette conférence ne sera qu'un moyen pour certains des participants de grimper les échelons académiques, d'obtenir une reconnaissance du gouvernement en échange de leurs idées sur comment gérer le "problème aborigène" et de s'assurer davantage de financements pour leurs recherches.

Il est peu probable, en revanche, que la conférence contribue à l'établissement d'une relation plus humaine entre les anthropologues et les personnes auprès desquelles ils tirent leur savoir (habituellement appelés "informateurs"). Il est peu probable également qu'elle les aide à comprendre les faits de la vie et de la mort aborigènes en Australie aujourd'hui. »

10 Cette idée que les universitaires feraient carrière "sur le dos" des Aborigènes, les dépossédant de leur « culture » à leur profit, revenait régulièrement au cours de la lettre. L'Institut se voyait ainsi accuser de fonctionner 
" comme une "confrérie" [fellowship] d'universitaires se soutenant mutuellement pour faire avancer leurs carrières, principalement en "sauvant" [salvaging from] les savoirs des Aborigènes, ainsi que leur propriété culturelle. »

11 La lettre insistait également à plusieurs reprises sur le fait que la motivation principale des membres de l'Institut aurait été « d'accroître leur influence sur le gouvernement dans sa gestion des affaires aborigènes », pris qu'ils étaient « dans une spirale de construction d'empire ».

Les militants dénonçaient ainsi la relation de servilité qui unissait les anthropologues au gouvernement, la définition des objets d'étude étant entièrement subordonnée à la volonté de plaire à ce dernier :

«Le moyen le plus efficace pour servir les désirs et les intérêts du gouvernement est de lancer et de financer des études dans les champs les plus pertinents que sont les changements et ajustements sociaux et la recherche destinée à influencer la politique gouvernementale. Ces tendances sont clairement perceptibles dans l'Institut d'aujourd'hui. »

\section{Les conditions de production du savoir anthropologique}

Un deuxième ensemble de critiques concernait la pratique de l'anthropologie elle-même. Au niveau de l'interaction individuelle tout d'abord, les signataires remarquaient que la « relation entre enquêteur et enquêté manquait d'humanité ». Des relations d'amitié, de confiance et d'honnêteté devaient être des conditions préalables à la recherche et à toute « relation inter-culturelle constructive [meaningful] ».

14 Leur critique des conditions de production du savoir anthropologique ne se limitait pas au seul niveau de l'interaction interpersonnelle. Pour les signataires, la relation d'enquête était également perturbée par des relations de pouvoir à un niveau plus large ; et il leur semblait nécessaire, pour ne pas produire un savoir décontextualisé, de tenir compte des relations structurelles de domination à l'échelle de la société. En témoignent ces deux passages :

"Ceux qui sont engagés dans les études aborigènes se trouvent nécessairement dans une situation sensible. Ils ne devraient pas prétendre réaliser des études objectives quand l'existence des Aborigènes est principalement caractérisée par l'oppression quotidienne venant du groupe auquel appartient, de plus ou moins loin, l'anthropologue.

La relation d'enquête peut finalement être résumée comme suit: un membre privilégié de la société opprimante étudie l'opprimé et donne des informations aux autres membres de la société opprimante et, dans de nombreux cas, à ceux qui sont les principaux "méchants" [villains] (gouvernement, intérêts miniers, industriels, pastoraux, immobiliers et touristiques). »

15 Prenant exemple sur le cas de Richard Gould, un anthropologue américain qui s'était impudemment affranchi de ses obligations de secret vis-à-vis de ses enquêtés ${ }^{11}$, les militants montrent que des relations de pouvoir et de distance prévalaient alors. Ils expliquaient que ce cas n'aurait pas dû être traité comme une exception, mais aurait dû permettre de repenser les relations entre les anthropologues, l'État et les Aborigènes. Il n'en a pas été ainsi :

"[L'affaire Richard Gould] touchait à des problèmes tout à fait centraux pour l'anthropologie, mais elle fut immédiatement requalifiée de "problème d'accès au terrain".

Comme par hasard [conveniently], les Aborigènes se sont retrouvés pris en sandwich dans une lutte de pouvoir entre, d'un côté, l'Institut, représentant les 
anthropologues et, de l'autre, les autorités gouvernementales, essayant d'étendre leur contrôle sur le système de permis pour l'entrée dans les réserves.

Aucun des plaignants n'était représenté à la rencontre organisée par l'Institut [...]. On considéra que Richard Gould était une exception. On fit de lui un bouc émissaire et la situation revint à la normale. C'est-à-dire que "l'accès au terrain" et aux "sujets d'enquête" fut restauré.

Quand un anthropologue suggéra à cette réunion que l'octroi de droits fonciers était la seule solution pour permettre aux groupes aborigènes d'accueillir ou d'exclure les visiteurs dans leurs propres termes, la solution fut rejetée comme irréaliste. »

\section{Un retrait du politique}

Ces critiques retombaient sur une prise de position plus ouvertement politique. On soulignera deux revendications majeures. D'une part, la nécessité de " prendre en compte l'existence et les intérêts des personnes étudiées [les populations aborigènes] ». D'autre part, celle de «la promotion sincère d'une forme d'Enlightenment dans l'Australie d'aujourd'hui ».

Len Watson en compagnie d'autres militants de Brisbane, Sam Watson S. et Tiga Bayles.

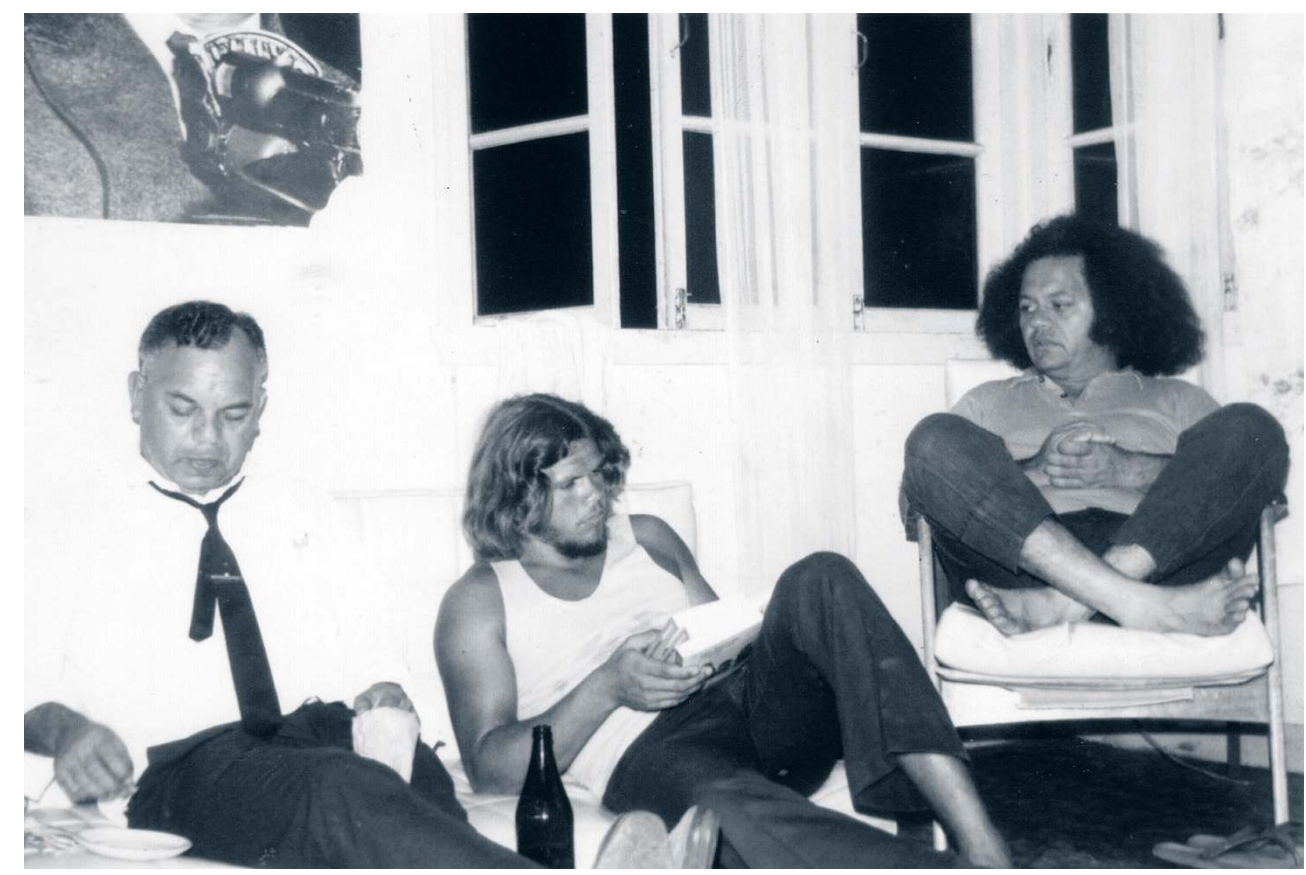

(c) Peter Thomson

En amont de la recherche, tout d'abord, les militants mettaient en question le choix des objets d'étude et les intérêts scientifiques des chercheurs, qui objectivaient les groupes aborigènes à titre de sujets d'expérience et négligeaient de s'occuper de leurs conditions d'existence contemporaines.

«Les universitaires devraient cesser de collecter des faits de plus en plus ésotériques qu'ils interprètent pour la consommation d'un public de plus en plus réduit. Leurs objectifs devraient être d'aider tous les gens, y compris ceux parmi lesquels ils vivent, à comprendre les caractéristiques générales et complexes de la situation australienne (colonialisme non résolu, capitalisme et privilège, autorité/ pouvoir).» 
L'idée était finalement que l'anthropologie ne servait à rien si elle ne pouvait pas être utilisée comme arme contre la situation de domination à laquelle se trouvaient confrontés les Aborigènes :

« Les anthropologues, de façon générale, ne contribuent pas à ce que les Aborigènes puissent comprendre, saisir et contrôler nos situations et nos destinées ${ }^{12}$. [...]

Quels efforts l'Institut a-t-il faits, malgré l'ensemble des données collectées, pour corriger les expressions implicites et explicites de supériorité culturelle et de racisme que l'on trouve dans les livres scolaires actuels, et qui semblent partagées par la majorité des Australiens?

L'Enlightenment dont nous parlons vise à faire comprendre aux Australiens que les pratiques culturelles aborigènes peuvent se poursuivre et se développer à la condition d'une sécurité économique et d'une liberté relative vis-à-vis de la persécution culturelle (blanche) et des préjugés. »

19 En aval de la recherche, les militants dénonçaient une instrumentalisation politique des recherches de l'Institut par le gouvernement australien dans une logique de délégitimation, au nom de la science, des revendications politiques aborigènes. On en retiendra deux exemples.

Les militants dénonçaient l'absence de soutien de l'Institut aux demandes de droits fonciers, soulignant que les anthropologues devaient «pourtant bien connaître l'importance de la terre pour les populations aborigènes ».

«Pour ne pas remettre en cause le système de valeurs de l'Australie blanche, le gouvernement évite absolument de se confronter aux vraies solutions. Les DROITS FONCIERS se voient ainsi discrédités au nom de la nécessité d'une "viabilité économique", et d'autres recherches visent à trouver des excuses pour ne pas donner aux Aborigènes indépendance et justice, ou à trouver des raisons prouvant que l'indépendance ne marcherait pas. »

21 De plus, ils remettaient en cause la division anthropologique entre "Aborigènes tribaux " et «détribalisés ». La prétention des membres de l'Eaglehawk and Crow à représenter l'ensemble des Aborigènes se voyait en effet délégitimée par le gouvernement et par certains anthropologues qui sous-entendaient qu'ils n'étaient pas de « vrais Aborigènes ». Les militants dénonçaient cette attitude comme doublement raciste. D'une part parce qu'elle créait une division arbitraire au sein de la population aborigène, d'autre part du fait de la condescendance vis-à-vis de ceux qui, justement, étaient qualifiés de «tribaux»:

«En janvier 1974, le nouveau directeur soulignait dans la Lettre de l'Institut que les relations avec les Aborigènes tribaux étaient bonnes. Il expliquait que la publication d'une brochure présentant l'Institut et ses objectifs dans diverses langues aborigènes devrait permettre d'améliorer encore ces relations.

Pourtant, tout n'est peut-être pas aussi rose qu'on voudrait nous le faire croire et la tactique selon laquelle l'usage des langues permet nécessairement communication et compréhension ne nous laisse pas dupes.

Les propres doutes de Peter Ucko apparaissent clairement dans la phrase suivante : "Espérons que la brochure puisse aussi contribuer à convaincre les Aborigènes non tribaux du bien-fondé des objectifs et de la fonction de l'institut." Veut-il dire par là que les Aborigènes qui savent lire et écrire l'anglais sont plus difficiles à convaincre de ses idées que ceux pour qui c'est plus difficile? Son patron, le sénateur Cavanagh ${ }^{13}$, avait tenu des propos similairement insultants en faisant remarquer la plus grande facilité à manipuler les Aborigènes qu'ils choisissent d'appeler "tribaux". ”

Face à cette menace, les militants tenaient au contraire à afficher l'unité des Aborigènes, quels que soient les États, et notamment l'unité face à l'oppression ${ }^{14}$. Ils réunissaient, par- 
delà la division "tribal/détribalisé » des anthropologues, les situations affectant les Aborigènes du nord de l'Australie (Arnhem ou Gove) et celles qui les touchaient plus directement (le Victoria, la Nouvelle-Galles du Sud et dans une certaine mesure le Queensland).

"L'oppression est un continuum qui affecte l'ensemble des Aborigènes: le tourisme, les projets d'exploitation forestière et minière en terre d'Arnhem, les activités minières à Gove et Weipa, l'aliénation des terres dans la NSW et le Victoria, et les Aborigines and Torres Strait Islander Acts dans le Queensland. »

\section{Les réponses}

La contestation ne laissa pas indifférent. Les militants reçurent dix-huit réponses provenant des deux directeurs successifs de l'AIAS, de chercheurs des plus grands départements d'anthropologie en Australie et d'anthropologues américains ayant fait du terrain en Australie. Le ministre des Affaires aborigènes lui-même y répondit ${ }^{15}$.

La plupart des chercheurs reconnaissaient que la lettre soulevait des points importants dont il fallait discuter. Même Richard Gould, pourtant ouvertement mis en cause dans la lettre, répondit: «Je suis certainement plus sensible à vos revendications que vous ne l'imaginez. »

25 Les chercheurs concédaient généralement que la situation de domination dans laquelle se trouvaient les Aborigènes posait problème. Certains appuyaient la critique de la «mentalité muséographique» de l'Institut ${ }^{16}$, d'autres insistaient sur la nécessité d'une représentation des Aborigènes en son sein, voire sur l'importance d'une reconnaissance des droits fonciers. Il faut se souvenir que l'Australie était dans un contexte de transformation de ses politiques aborigènes - l'arrivée de Gough Whitlam au pouvoir en 1972 marquait l'abandon officiel de la politique «d'assimilation» pour une politique "d'autodétermination ${ }^{17}$ » - et que toute opposition franche au leadership aborigène émergent pouvait être comprise comme une complicité avec "l'ancien régime ${ }^{18}$ ", avec lequel l'establishment anthropologique avait travaillé en association étroite. A.P. Elkin, figure dominante de l'anthropologie australienne des années 1930 au début des années 1960, fut notamment l'un des artisans de la politique d'assimilation et il était un des responsables du New South Wales Aboriginal Welfare Board.

Néanmoins, le soutien apporté par les anthropologues à la lettre doit être nuancé.

Tout d'abord, certains chercheurs utilisèrent cette lettre à leur profit dans la lutte qu'ils menaient à l'intérieur du champ anthropologique. Ainsi, T.G.H. Strehlow, de l'Université d'Adélaïde, en conflit avec l'AIAS depuis quelques années, appuyait fortement l'attaque lancée par le groupe et n'hésitait pas à y ajouter ses propres critiques. Il adressa d'ailleurs à l'Eaglehawk and Crow une copie de la lettre de démission qu'il avait envoyée à l'Institut une année auparavant. Pourtant, si sa lettre rejoignait certaines critiques des militants aborigènes, notamment la dénonciation de la "dépossession » des Aborigènes par les anthropologues, son "alliance» avec les militants du sud-est de l'Australie ne l'empêchait pas, par ailleurs, de fustiger ceux qu'il ne considérait pas comme « de vrais Aborigènes » et il lui arrivait de renvoyer dos à dos les « anthropologues mal formés » et leurs «associés blancs ou moitié blancs ${ }^{19}$ ». En ce sens, Strehlow revendiquait également pour lui-même le monopole de la représentation légitime de la « nature authentique » des populations aborigènes. 

Aborigènes - comme eux, elle condamnait les conceptions anthropologiques fixistes prévalant à l'Institut - et elle profitait de sa lettre pour demander à ces derniers leur soutien au développement du centre qu'elle dirigeait à Monash University. Celui-ci serait plus démocratique et plus ancré dans le présent que l'AIAS.

Lyn Thomson (née Craigie) et Terry Widders ont participé en décembre 1972 au voyage de la première délégation aborigène invitée en Chine. Lyn ( $2^{\mathrm{e}}$ à partir de la gauche) ici photographiée avec trois autres militants (de g. à d. Chicka Dixon, Ruby Hammond et Gerry Bostock) lors d'une conférence de presse au retour du voyage.

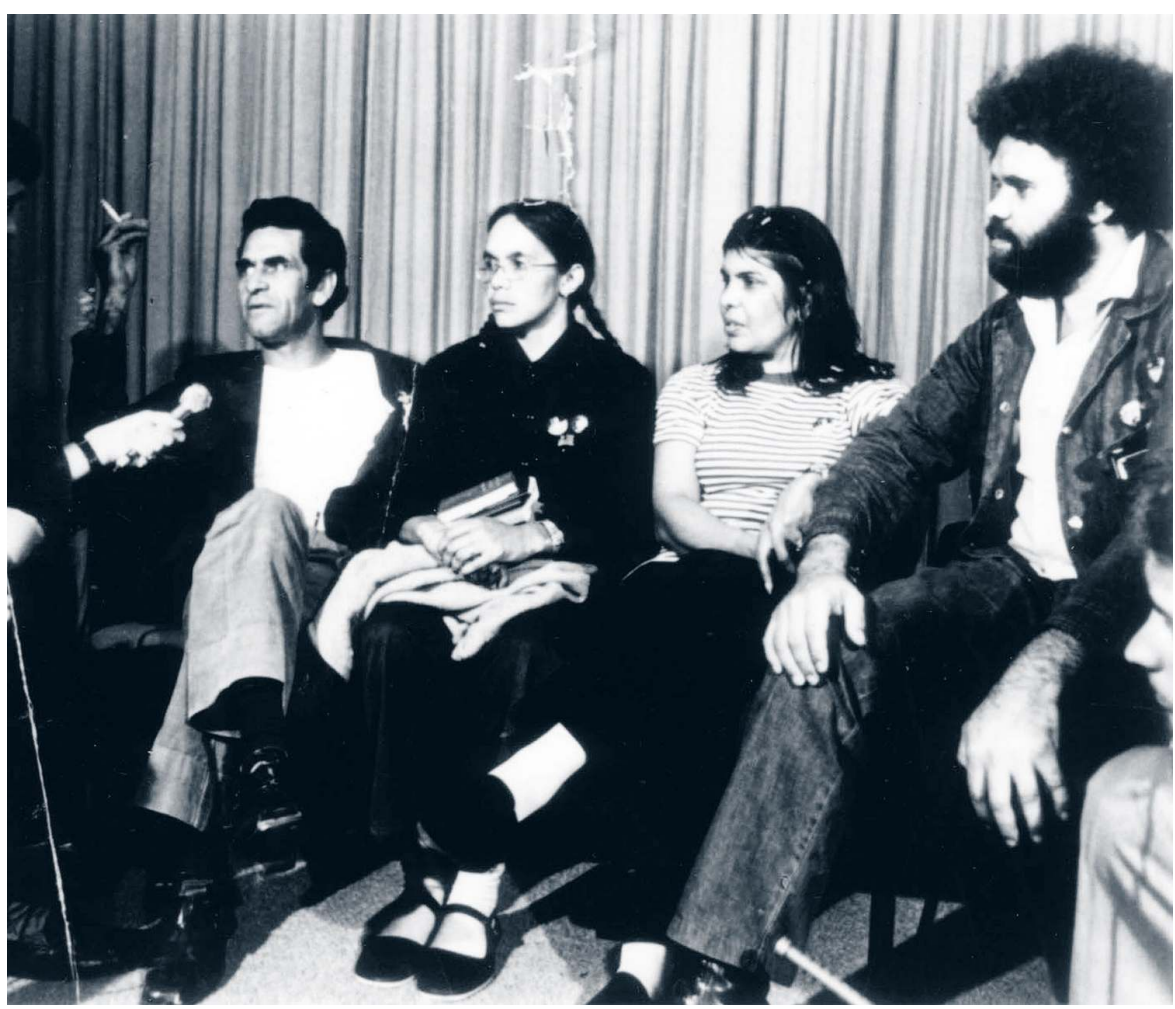

(c) Peter Thomson

De plus, si les anthropologues reconnaissaient certaines erreurs commises au sein de la profession (principalement liées au vol d'objets et au non-respect du secret), ils refusaient généralement d'admettre l'existence d'un problème structurel. Leur argumentaire peut être résumé ainsi : nous reconnaissons que l'histoire de l'anthropologie n'est pas sans pages noires, mais les «dérapages » sont essentiellement le fait d'une minorité, et nous n'avons personnellement pas grand-chose à nous reprocher. Même les chercheurs affichant une certaine sympathie pour la lettre laissaient entendre que ses auteurs avaient tendance à grossir le trait. Ils pensaient que, d'une part, les anthropologues n'avaient pas autant de pouvoir qu'on voulait le faire croire (J.W. Warbuton) et que, d'autre part, tous ne tombaient pas dans les travers dénoncés (Derek Freeman, Elisabeth Eggleston). R.G. Hausfeld, de l'Université de Sydney, affirmait ainsi que « depuis 25 ans qu'il était anthropologue, il ne se sentait pas investi d'un pouvoir particulier » et qu'il "était aussi injuste d'accuser tous les anthropologues de telles malversations [déplacement d'objets, révélation d'informations devant rester secrètes, mauvais comportements sur le terrain, etc.] que d'accuser tous les Aborigènes d'être des ivrognes et des paresseux ». Il soulignait que le travail demandé aux chercheurs n'était pas facile et 
souvent ingrat, remarquant qu'il aurait très bien pu, dans ses enquêtes sur la pauvreté, ne pas travailler sur les Aborigènes, « ce qui [lui] aurait facilité bien des choses ». Warbuton ajoutait, quant à lui, que certains anthropologues, comme Elkin et Berndt, avaient largement contribué au bien-être des Aborigènes, "à une époque où ce n'était décidément pas une cause populaire ». Par ailleurs, certains chercheurs soulignaient que l'Institut n'était peut-être pas une institution parfaite mais qu'il avait au moins le mérite de permettre à des gens de travailler sérieusement sur la question aborigène. Ainsi Isobel White, de Monash University, constatait simplement: "Sans les financements de l'Institut, je n'aurais pas pu faire de terrain avec certains groupes aborigènes et je ne saurais rien des Aborigènes ni de leur culture traditionnelle, de leur mode de vie actuel, de leurs problèmes présents sous la domination blanche.»

Enfin, pour Hausfeld, du fait même de la mission qui lui avait été fixée, l'Institut se devait de rester en dehors de la politique. De même, Jack Golson de l'Australian National University pensait que les opinions privées des chercheurs ne devaient pas engager l'AIAS. Il justifiait sa position au moyen d'une comparaison: «Appartenant au O'Connor Cricket Club, me serait-il légitime de demander aux autres membres de protester, au nom de l'organisation, contre le renversement d'Allende par les militaires chiliens? »

Bob Bellear, l'un des organisateurs de la « tente ambassade ", deviendra plus tard le premier juge aborigène.

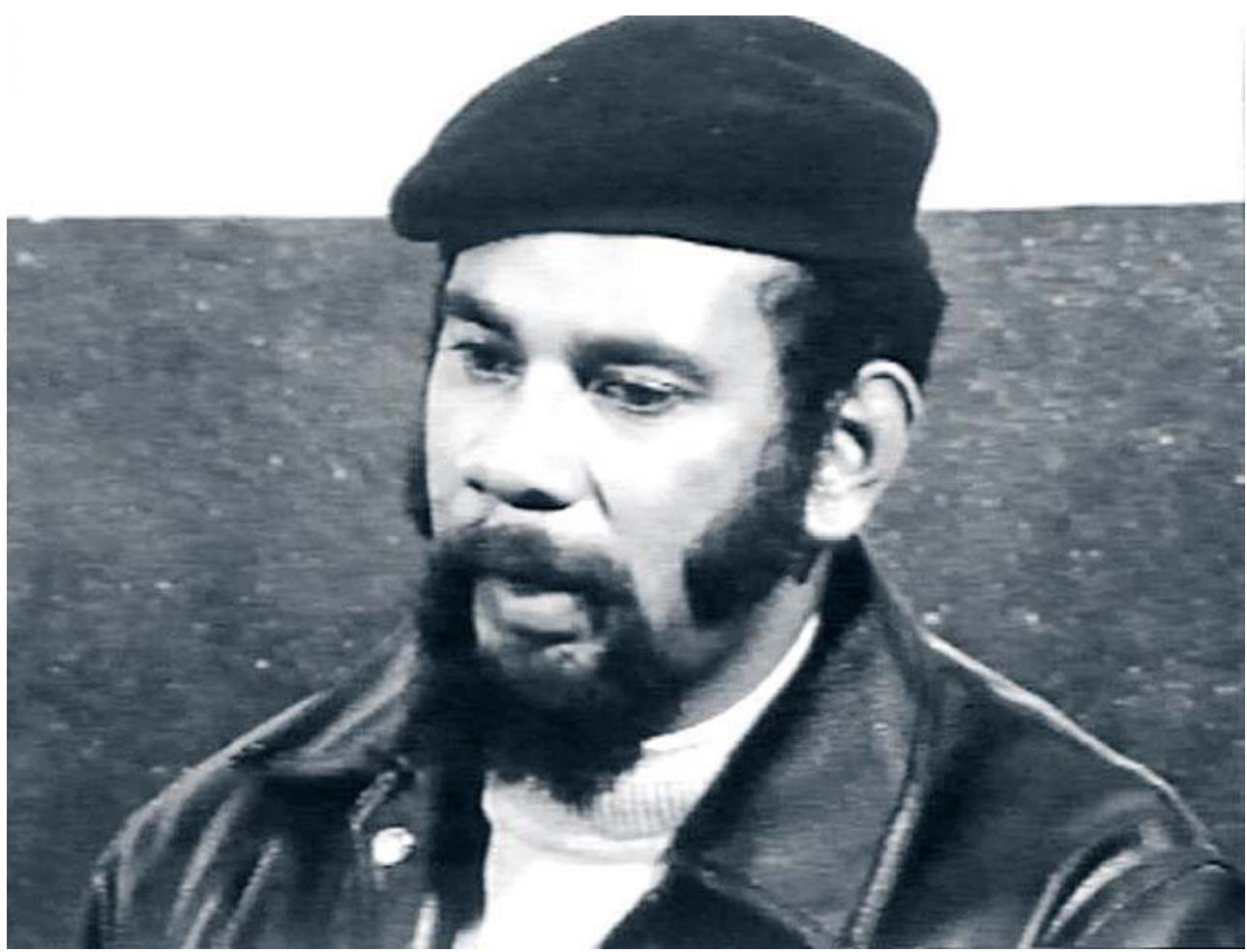

(c) Peter Thomson

Quelques lettres, provenant essentiellement de chercheurs appartenant à la «vieille garde » des études aborigènes, étaient ouvertement hostiles à la démarche de l'Eaglehawk and $\mathrm{Crow}^{20}$. Celle de Fred McCarthy, premier directeur de l'AIAS, nous paraît tout à fait exemplaire. Il y expliquait que la recherche académique pure faisait «intégralement partie de notre culture blanche ou d'Europe occidentale » et qu'il n'était donc ni utile ni souhaitable de « sacrifier la recherche culturelle pure » pour « faire couler l'argent à flots 
dans les activités aborigènes ${ }^{21}$ ». Le coût de la conférence, expliquait-il, était infime lorsqu'on le comparait "aux millions de dollars dépensés pour le welfare aborigène au cours des cent dernières années». Il ajoutait que si les "anthropologues blancs» n'avaient pas réalisé ce travail de "sauvetage", personne ne l'aurait fait et les informations auraient été perdues à jamais. Isobel White, pourtant plus sensible à la lettre, l'appuyait sur ce point. « Sans le travail, certes parfois indigne et égoïste, accompli jusqu'à aujourd'hui par les chercheurs blancs, la plupart des traditions aborigènes auraient disparu. [...] C'est grâce aux enregistrements réalisés par quelques Blancs bienveillants que vous avez vous-mêmes quelques connaissances des traditions de vos ancêtres » (c'était aussi l'argument d'Arnold Pilling).

McCarthy allait plus loin en expliquant que les anthropologues avaient souvent essayé de former des Aborigènes pour qu'ils les assistent dans leur travail, mais que leur sollicitation débouchait généralement sur un refus. «Combien d'Aborigènes urbains connaissent un tant soit peu la culture de leur peuple? Combien s'y intéressent véritablement? ironisait-il. Parce qu'ils sont obsédés par les revendications de droits fonciers et le contrôle de l'argent public, ils négligent l'aspect culturel de la vie aborigène. C'est donc aux anthropologues blancs qu'il revient de l'enregistrer. » Il ajoutait avec une naïveté feinte que «rien n'empêch[ait] les Aborigènes de devenir anthropologues et d'écrire des livres comme ceux de Berndt ou d'Elkin pour leur propre profit. La recherche anthropologique et l'écriture demandent simplement de l'acharnement au travail ». Renvoyant la responsabilité sur les Aborigènes eux-mêmes, il arguait que « c'était à eux de s'élever socialement, d'accéder à une certaine éducation, de combattre les problèmes d'alcoolisme et de prostitution minant leurs communautés ».

Nous retrouvons clairement ici le conflit mis en avant par Benoît de L'Éstoile entre, d'un côté, la «légitimité esthético-scientifique de l'ethnologue qui revendique un accès privilégié à la "civilisation indigène" authentique et s'en fait l'interprète devant les Européens » et, de l'autre, la « légitimité politique » de l'intellectuel/militant indigène ${ }^{22}$.

\section{Le texte à la lumière des trajectoires militantes}

Plusieurs lectures des critiques présentées par les militants aborigènes sont possibles. Il nous paraît important, au préalable, de présenter brièvement les trajectoires et les histoires sociales individuelles des rédacteurs de la lettre. Enfants du baby-boom, ils ont grandi tiraillés par des forces contradictoires pendant la politique d'assimilation ${ }^{23}$ progressivement mise en place après la Seconde Guerre mondiale. Ce sont ces contradictions inscrites dans leurs histoires sociales personnelles, construites sur une opposition entre les deux instances principales de socialisation, la famille et l'école, qui permettent de saisir le rapport particulier qu'ils ont à l'anthropologie.

D'un côté, ces militants ont grandi dans des milieux aborigènes très autonomes. Les entretiens que nous avons menés et les archives que nous avons consultées indiquent que les Aborigènes vivaient à cette époque de façon très indépendante par rapport à "l'Australie blanche ${ }^{24}$ ", principalement dans les réserves du gouvernement, dans des bidonvilles sur le bord des rivières ou à la lisière des villes. Les rapports annuels de l'Aboriginal Welfare Board (AWB), organisme chargé de l'administration et de l'assimilation des Aborigènes de Nouvelle-Galles du Sud, en témoignent. Le Board 
soulignait par exemple dans son rapport pour 1952 les difficultés de ses agents à assimiler les populations aborigènes :

«C'est cette déférence pour le passé et la détermination qu'ils ont à rester ensemble, ainsi peut-être que les efforts de la population blanche pour les tenir à

l'écart, qui constituent les principaux obstacles au travail du Welfare Officer ${ }^{25}$. ”

Pour autant, les communautés aborigènes n'avaient pas conservé un mode de vie identique à la période pré-coloniale. La colonisation à laquelle elles avaient été confrontées depuis la seconde moitié du XIX ${ }^{e}$ siècle les avait profondément affectées. Gary Williams et Terry Widders m'expliquaient ainsi qu'ils avaient grandi « dans l'ombre » de «l'ancienne culture », puisque c'était la génération de leurs grands-parents qui avait connu les dernières initiations ${ }^{26}$. Néanmoins cette quasi-séparation du reste de l'Australie a joué un rôle important de protection vis-à-vis des pressions imposées par «l'Australie blanche » et offrait, semble-t-il, un « quant-à-soi » assez rassurant ${ }^{27}$.

De l'autre côté, ces militants sont les enfants de la politique d'assimilation. Tous ont bénéficié, dans leur jeunesse, de bourses du gouvernement australien ou d'organisations caritatives et faisaient partie d'une toute petite minorité d'enfants aborigènes considérés comme des modèles par l'AWB ${ }^{28}$. Ils apparaissaient plus ou moins régulièrement dans Dawn, le magasine créé par le Board et distribué dans les communautés aborigènes pour contribuer, grâce à des articles "éducatifs et instructifs ", à la politique d'assimilation. Bob Bellear reçut ainsi une bourse de l'AWB pour aller au lycée, il fut élu capitaine de l'équipe de football en 1960 puis "préfet» de son école en 1961. Lyn Thomson (née Craigie) fit des études à l'Armidale High School puis suivit une formation de commerce à l'East Sydney Technical College. Gary Williams, après des études secondaires dans un lycée catholique huppé, fut le premier étudiant aborigène reçu à l'Université de Sydney ${ }^{29}$. Terry Widders obtint lui aussi une bourse qui lui permit de passer quatre années dans l'internat d'un lycée bourgeois tenu à Armidale par la Church of England. Il interrompit néanmoins sa scolarité quelques mois avant les examens finaux, préférant aller «travailler avec ses cousins »:

« Pour eux c'était normal de quitter l'école vers 14/15 ans et d'aller travailler... Ils avaient de l'argent, ils avaient des petites copines. J'étais jaloux... J'étais coincé dans ce système. »

Cette dernière citation montre combien leurs parcours étaient singuliers à une époque où la majeure partie des Aborigènes interrompaient leur scolarité très tôt. L'AWBreconnaissait ainsi dans ses rapports sa grande difficulté à scolariser les enfants aborigènes ${ }^{30}$. En 1961, à peine six enfants aborigènes étaient arrivés au leaving certificate.

Au cours des années 1960, à la sortie de l'adolescence, ces militants prirent part à un mouvement d'immigration vers les grandes villes (Sydney, Melbourne, Brisbane) qui touchait massivement les communautés aborigènes suite au démantèlement des réserves. C'est à Sydney, dans le cadre de la Foundation for Aboriginal Affairs, association qui servait de centre social pour les Aborigènes, qu'ils se rencontrèrent. Avec d'autres Aborigènes de leur âge, ils formèrent très rapidement un groupe soudé. Leur éducation politique se fit au contact de militants aborigènes plus âgés, notamment le syndicaliste Chicka Dixon et l'ancien joueur de football Charlie Perkins, mais aussi, de façon plus pragmatique, en réaction au harcèlement policier qui sévissait à Redfern, le quartier aborigène de Sydney ${ }^{31}$. En quelques années, ils devinrent les figures dominantes du mouvement aborigène, stigmatisant les militants des générations antérieures qu'ils considéraient comme insuffisamment revendicatifs. S'inspirant assez largement du 
militantisme noir américain, ils imposèrent un langage nouveau - Black control of Black affairs - et se lancèrent dans une entreprise de face-à-face avec «l'Australie blanche ». Ils organisèrent de nombreuses actions symboliques et autres manifestations de rue ${ }^{32}$, contribuèrent à la prise de contrôle par les Aborigènes eux-mêmes des associations qui travaillaient pour "l'avancement des Aborigènes ${ }^{33}$ " et créèrent des organisations de « survie communautaire» (Legal Service, Medical Service, Housing Company, etc.). Au moment de la lettre, les signataires faisaient donc partie d'un groupe de militants occupant le devant de la scène politique australienne ${ }^{34}$.

Jusqu'où allait, à travers cette lettre, leur incursion dans l'univers anthropologique et quelles étaient leurs prétentions?

Une première interprétation possible consiste à prêter aux Aborigènes un point de vue que je qualifierais de strictement anti-scientifique. Plusieurs éléments permettent, à première vue, de soutenir cette hypothèse.

On remarquera tout d'abord que la critique de l'anthropologie par les Aborigènes était, pour une large part, une importation dans le champ scientifique de la critique radicale qu'ils formulaient dans le champ politique. Or cette extension à l'univers de la science de l'idée d'un contrôle aborigène des affaires aborigènes revenait à contester la légitimité des non-Aborigènes à produire un savoir sur les Aborigènes. D'une certaine façon, les «Blancs » étaient forcément du côté des oppresseurs, ce qui disqualifiait a priori toute prétention à produire un savoir un tant soit peu objectif.

Un des arguments forts du texte est en effet qu'aucune « connaissance objective » n'était possible dans le contexte de domination caractérisant la situation australienne. Ce qui revient nécessairement à subordonner l'exigence de vérité à la visée d'émancipation. La seule science utile, et donc possible, devait servir l'avancement de leur cause.

Dans cette optique, la lettre et les tensions entre ce groupe de militants aborigènes et la profession anthropologique peuvent être comprises comme un conflit autour du droit à la représentation légitime des Aborigènes. En quelque sorte, la lettre correspondait à une lutte pour des ressources rares et renvoyait à une question simple : à qui doit revenir le contrôle de l'argent versé dans les affaires aborigènes ${ }^{35}$ ? Les militants exprimaient leur frustration en soulignant que l'argent devrait prioritairement aller aux communautés elles-mêmes au lieu d'être accaparé pour des études dont ils ne voyaient pas l'intérêt et sur lesquelles ils n'avaient pas prise.

«L'argent et les autres ressources font défaut quand il s'agit du contrôle par les Aborigènes de leur propre existence, mais il ne manque pas, semble-t-il, lorsqu'il s'agit d'en discuter. »

45 Par ailleurs, les militants aborigènes ne dénonçaient pas seulement le pouvoir d'influence des anthropologues sur le gouvernement (qui leur permettait, on l'aura compris, de s'assurer un soutien financier en retour), ils critiquaient aussi leur capacité à peser sur la définition même de l'identité aborigène :

« Du fait de l'éclatement partiel de la tradition orale aborigène, [...] le savoir issu des livres devient la référence de l'aboriginalité [aboriginality, c'est-à-dire "l'essence aborigène"]. Les anthropologues exercent donc leur pouvoir à la fois sur la pensée aborigène et sur la politique du gouvernement par rapport aux besoins et sentiments des Aborigènes. »

Une seconde lecture consiste à considérer que ce n'est pas la possibilité de faire la science elle-même qui était mise en question mais les conditions actuelles de sa pratique. Selon 
cette interprétation, le point de vue des militants aborigènes était plus " anti-scientiste " que véritablement « anti-scientifique ».

Suivant une logique de compromis, leur ambition n'était pas "d'interdire la science ", mais, au contraire, de contribuer à la rendre plus pertinente. D'où les remarques, que nous avons soulignées, qui interpellaient différentes facettes de la pratique scientifique (ce ne sont pas les bonnes questions qui sont posées, la relation d'enquête n'est pas pertinente, il faut, sinon rééquilibrer, du moins tenir compte des relations de pouvoir, etc.) et dénonçaient comme illusoire l'idée de "neutralité de la science » en faisant remarquer l'instrumentalisation politique par le gouvernement australien des travaux des chercheurs.

Deux remarques de la lettre témoignent d'ailleurs d'un intérêt et d'une volonté de leur part d'être impliqués dans le projet de connaissance du monde aborigène ${ }^{36}$, et de ce qu'ils percevaient comme une relative indifférence de l'Institut à leur égard :

«Deux d'entre nous, en 1972 et à nouveau en 1973, approchèrent l'Institut, demandant que soient rendus accessibles aux communautés de la côte nord de la Nouvelle-Galles du Sud des matériaux de l'Institut sur les dialectes bandjalang ${ }^{37}$. Nous ne reçûmes aucune réponse, ni de l'ancien directeur, ni du nouveau.

L'un d'entre nous, en réponse à un questionnaire que l'Institut avait fait circuler pour préparer la conférence de mai, suggéra que trois d'entre nous puissent présenter des exposés au séminaire sur la question du "Changement culturel et social". L'Institut estime apparemment que les Aborigènes qui sont au cœur de l'action et de la compréhension du changement ne sont pas spécialement compétents pour en parler. Ils ne furent pas invités, l'Institut se contentant de leur envoyer un questionnaire. »

De même, ils ne revendiquaient pas tant un contrôle total des ressources que le droit à une participation réelle à l'intérieur du système et ils dénonçaient l'exclusion dont ils s'estimaient victimes :

« Nous ne voulons pas d'une coopération seulement pour la forme [tokenism]. Philip Roberts $^{38}$ en a suffisamment souffert. Le comité "Site of Significance" de l'Institut devra comporter "au moins un Aborigène pleinement initié". Cette personne risque d'en souffrir également.

En 1970, un sous-comité de l'Institut recommanda que des Aborigènes puissent devenir membres associés et que des comités aborigènes soient créés pour proposer des lignes de recherche. Même ces réformes timides, et aujourd'hui totalement inadéquates, n'ont jamais été acceptées par l'Institut. »

Il est néanmoins difficile de préjuger a priori des intentions des militants. Leur texte n'est pas un traité d'épistémologie, mais un document politique qui, indéniablement, joue avec les ambiguïtés et soutient des positions parfois contradictoires. Pour comprendre ces contradictions, plutôt que de proposer une analyse «interne» ou «intellectualocentrée ", nous avons voulu mettre la lettre en relation à la fois avec les trajectoires biographiques des militants, que nous avons présentées rapidement, et avec la position qu'ils occupaient dans la structure des champs politique et scientifique à cette époque. 


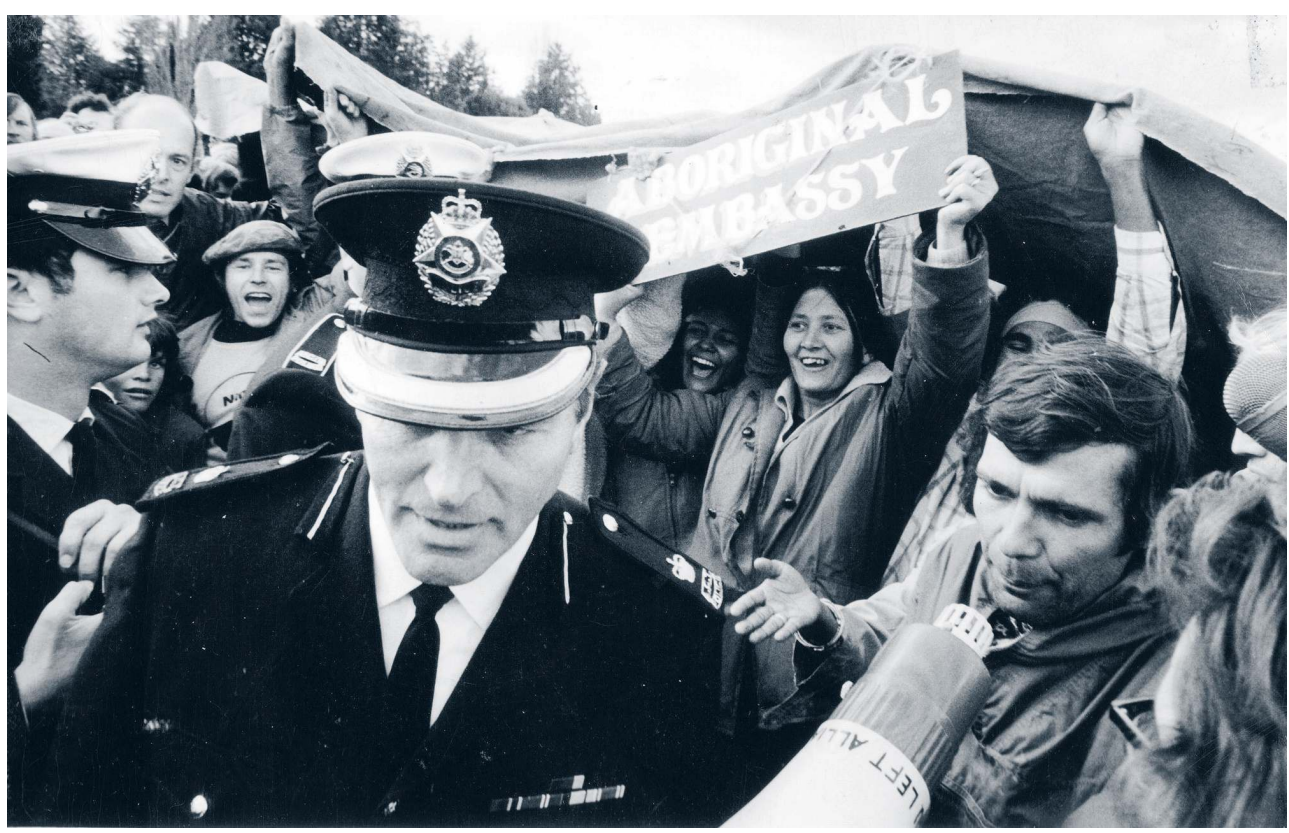

(C) Peter Thomson

Le rapport particulier de ces militants à la culture scolaire mérite tout d'abord d'être interrogé. D'un côté, il est fort probable que, étant donné leur trajectoire scolaire, leur rapport à la science n'était pas marqué par un anti-intellectualisme fort: leur passé de «bons élèves » a probablement développé chez eux une certaine croyance en l'autorité sociale et intellectuelle de la science, un certain respect pour l'école. D'un autre côté, le fait qu'aucun d'entre eux n'ait eu d'expérience effectivement réussie au sein de l'institution scolaire et universitaire montre qu'ils n'étaient pas dans un rapport de familiarité complète avec celle-ci. Ceux qui s'étaient confrontés aux premiers cycles universitaires (Gary Williams s'était réinscrit à la faculté de droit en 1971 et Terry Widders avait commencé une formation préparant à l'enseignement en 1972) avaient rencontré des difficultés et avaient rapidement interrompu leurs études.

La position qu'ils occupaient dans les champs politique et intellectuel confirme cette bivalence. Certains éléments témoignent ainsi de leur proximité relative avec le monde universitaire au moment de leur entrée en politique. Ils avaient par exemple établi des relations étroites avec certaines sections de la population étudiante (notamment ses franges les plus radicales). Dans un contexte d'intense agitation politique, alors que les campus étaient des lieux de débats permanents, les manifestations contre la guerre du Viêtnam et contre l'apartheid ont joué un rôle majeur dans la socialisation politique des militants aborigènes. Les activités politiques en commun avec des étudiants leur permirent de se lier d'amitié avec des gens comme Meredith Burgmann ${ }^{39}$, responsable de la lutte anti-apartheid à Sydney, ou Richard Refshauge, étudiant à l'ANU, qui a organisé l'aide des étudiants à l'ambassade aborigène.

53 Pour autant, aucun n'avait alors la prétention d'entrer dans le champ universitaire : leurs ambitions étaient ailleurs. Leur action s'inscrivait dans le monde politique et au sein des organisations qu'ils avaient contribué à créer et où une place de choix leur était réservée. Gary Williams et Lyn Thomson travaillaient pour l'Aboriginal Legal Service, Bob Bellear 
pour l'Aboriginal Housing Company, Terry Widders pour l'Aboriginal Arts Board et le World Council of Church. Le contraste était alors trop important entre le peu de ressources dont ils disposaient, en l'état du champ, pour pouvoir aspirer à des carrières académiques et les atouts dont ils bénéficiaient pour travailler sur le front de l'agitation politique, où le contexte politique leur garantissait un accès facile et rapide à des postes intéressants: le gouvernement Whitlam finançait les affaires aborigènes et les organisations qu'ils géraient en étaient des bénéficiaires importants. Même Peter Thomson, malgré sa formation universitaire et sa légitimité potentielle dans le milieu, ne se pensait pas comme un prétendant au monde académique et préférait travailler en contact avec ces mêmes organisations ${ }^{40}$.

Enfin, une attention aux trajectoires qui ont été les leurs postérieurement à la lettre confirme ce rapport équivoque. Ainsi, Terry Widders est entré à l'université en 1976 et y est resté depuis. Il faut dire que le rapport de force s'est ensuite inversé : alors que les financements gouvernementaux en direction des organisations aborigènes se sont taris, les opportunités pour entamer des études universitaires se sont multipliées grâce aux politiques de discrimination positive. Néanmoins, Terry Widders explique assez clairement le malaise qu'il ressent dans le milieu.

«Je ne suis pas un "académique"... Je te dis ça sans jouer au plus malin... Si je

pouvais, je ferais autre chose... Mais je ne vois pas bien ce que je pourrais faire. »

Inversement, Gary Williams est resté en dehors du milieu universitaire, mais il a continué à s'intéresser aux sociétés aborigènes. Il a notamment été très impliqué dans un projet visant à retrouver les langues de la région où il a grandi et où il vit aujourd'hui.

\section{Les effets du texte}

Nous aimerions conclure en nous posant la question des effets du texte, par-delà les intentions de ses auteurs.

Plusieurs éléments indiquent que la lettre ne fut pas sans conséquence sur le développement de l'anthropologie australienne ${ }^{41}$. La revue des activités de l'Institut pour l'année 1974 par son président, Peter Ucko, en est un premier indice. Ce dernier, sans pour autant mentionner la lettre, déclarait :

«Le principal changement, et celui qui m'a, personnellement, apporté le plus de satisfaction, est sans conteste la décision du conseil de suspendre certaines règles de l'Institut de façon à ouvrir les adhésions et permettre d'inclure des Aborigènes qui ont rendu des services aux études aborigènes, en plus des universitaires (à la fois aborigènes et non aborigènes). »

Le contraste tout à fait saisissant entre la sérénité affichée ici et la crispation suscitée par la lettre invite évidemment à ne pas prendre au premier degré cette déclaration. Nick Peterson, qui était alors en début de carrière, confirme l'influence du texte. Dans son allocution pour la Wentworth Lecture de 1990, il soulignait que cette protestation avait marqué l'émergence d'une phase nouvelle dans l'histoire australienne de la discipline, celle d'une " anthropologie négociée ${ }^{42}$ ».

«La lettre était remarquable pour son temps et contribua à précipiter des changements dans la gestion de l'AIAS, même si ceux-ci ne se firent que très progressivement. " 

discipline est généralement minimisé. En témoigne la version de Ronald Berndt qui, dans un texte revenant sur les grandes évolutions de l'anthropologie aboriginaliste, nie tout aspect conflictuel et tout rapport de domination entre enquêteurs et enquêtés. Il présente les changements intervenus comme s'inscrivant dans l'évolution naturelle de la discipline :

«En 1974, l'Institut se préparait à accepter des adhésions aborigènes. D’une certaine façon, cela correspondait à ce que les anthropologues avaient toujours fait sur le terrain. Au moins dans l'idéal, les anthropologues ont l'habitude de travailler en association très proche avec les Aborigènes, construisant et maintenant des relations interpersonnelles et considérant généralement leur recherche comme coopérative ${ }^{46}$.»

Il semble pourtant que l'anthropologie, comme science, n'a pas uniquement évolué à la suite de débats entre anthropologues, mais également du fait d'une opposition venant d'agents extérieurs à la discipline et notamment de certaines fractions des populations colonisées ${ }^{47}$. Dans certains contextes, et c'est ici le cas, les remises en cause entraînées, directement ou non, par les oppositions anti-coloniales ne se situaient pas uniquement à la marge de la discipline, mais concernaient son objet même et sa raison d'être ${ }^{48}$. 
Au premier plan, la tente de l'ambassade aborigène, fondée en 1972, comme acte politique majeur de l'histoire politique aborigène.

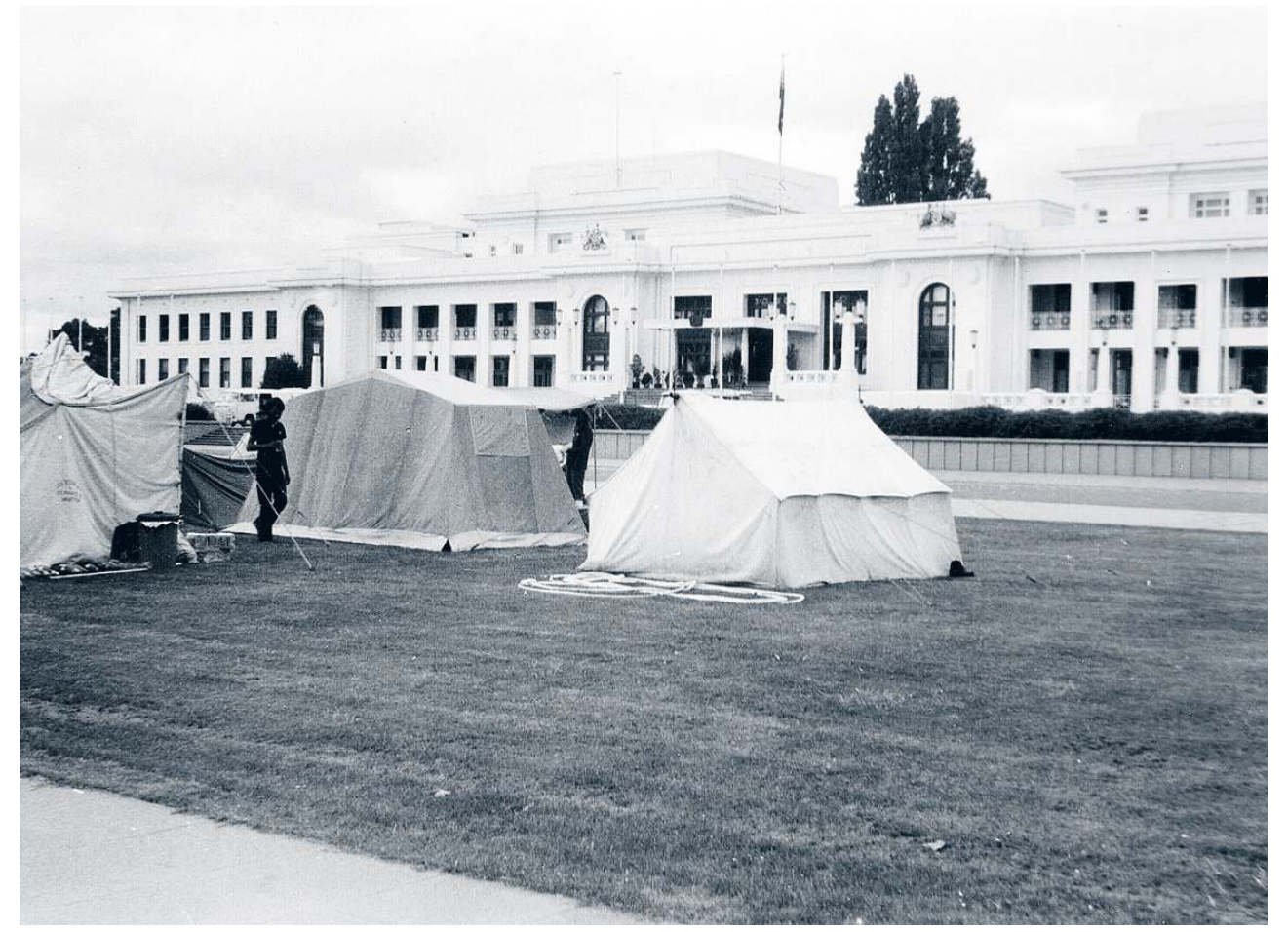

(c) Peter Thomson On voudrait plutôt suggérer une piste de recherche et un déplacement du regard. Cet «oubli» de la contestation anti-coloniale de l'anthropologie a partie liée au fait que, d'une part, les sources écrites sont rares et que, d'autre part, l'opposition des colonisés ne bénéficie généralement d'aucun ancrage institutionnel et se trouve donc rapidement marginalisée. Si le degré de verrouillage des frontières d'une discipline est variable selon les contextes, il semble que la routinisation et l'institutionnalisation des pratiques qui s'opèrent après une période d'ouverture permettent, à travers la formation d'une nouvelle doxa, une amnésie et une réappropriation des transformations par les tenants de cette doxa. Nous l'avons montré, la lettre n'était pas simplement le symptôme d'un affrontement entre deux univers distincts mais faisait aussi écho à des enjeux internes à l'univers des anthropologues. Certains, à l'intérieur même du monde universitaire, en appelaient ainsi à des redéfinitions de la discipline (quitte à revendiquer une part d'engagement ou de militantisme) contre des concurrents fidèles au dogmatisme légitime ${ }^{51}$. De ce fait, il n'est pas certain que, au bout du compte, l'autonomie du champ 
scientifique soit sortie véritablement affaiblie du fait de cette confrontation. Pour l'essentiel, les scientifiques sont restés des scientifiques et les militants des militants ${ }^{52}$.

Ce texte est donc relativement déconcertant. Il peut être compris comme l'entrée paradoxale dans le champ scientifique d'idées portées par des agents qui n'y avaient pas d'aspirations directes. Nous l'avons dit, les militants aborigènes n'avaient ni les capitaux scientifiques incorporés ni les dispositions pour se poser en nouveaux entrants. Étant donné les opportunités dont ils disposaient par ailleurs, le droit d'entrée était trop élevé et ne valait pas l'investissement.

Cette lettre permet ainsi de poser empiriquement la question théorique de la frontière des champs. On voit bien qu'il n'est pas nécessaire que des agents appartiennent strictement à un champ (au sens de la participation à la lutte entre dominants et prétendants) pour que non seulement ce qui s'y déroule affecte leurs intérêts, mais aussi, et c'est plus rare, pour qu'ils en aient conscience et souhaitent y accomplir quelque chose. $\mathrm{Du}$ fait d'un contexte très particulier ${ }^{53}$, les militants aborigènes, pourtant largement situés hors champ, escomptaient ainsi percevoir des profits politiques d'un changement de rapport de force à l'intérieur du champ scientifique. Ceci notamment parce que l'objet même de la lutte interne pour l'autorité scientifique dans le champ anthropologique - le pouvoir de produire, d'imposer et d'inculquer la représentation légitime des Aborigènes était un enjeu de la lutte qu'eux-mêmes menaient dans le champ politique.

\section{BIBLIOGRAPHIE}

BERNDT, Ronald

1982 « The Changing face of Aboriginal Studies : some personal glimpses », in G. McCall, Anthropology in Australia ; essays to honour 50 years of Mankind. Sydney, Anthropological Society of New South Wales : 49-65.

BOURDIEU, Pierre

1976 « Le champ scientifique », Actes de la Recherche en Sciences Sociales 2/3 : 88-104.

COOPER, Frederick et Stoler, ANN Laura éd.

1997 Tensions of Empire, Colonial Culture in a Bourgeois World. Berkeley, University of California Press.

GAÏTI, Brigitte

2002 « La science dans la mêlée : usage croisé des discours savants et militants », in Philippe Hammam, Jean-Matthieu Tieon et Benoît Verrier, Discours savants, discours militants. Paris, L'Harmattan : 293-309.

GLOWCZEWSKI, Barbara 
2004 Rêves en colère. Avec les Aborigènes australiens. Paris, Plon (« Terre humaine »).

GOODALL, Heather

1996 Invasion to Embassy. Sydney, Allen and Unwin.

HAMILTON, Anette

1982 « Anthropology in Australia : some notes and a few queries », in G. McCall, Anthropology in Australia; essays to honour 50 years of Mankind. Sydney, Anthropological Society of New South

Wales : 91-106.

L'ESTOILE (de), Benoît

1997 « Au nom des “vrais Africains” », Terrain 28 « Miroirs du colonialisme » : 87-102.

MoIzo, Bernard

1997 «L'anthropologie aboriginaliste : de l'application à la fiction », in Michel Agier, Anthropologues en danger. Paris, Jean-Michel Place : 65-74.

PETERSON, Nicholas

1990 « Studying Man and Man's nature », The History of Institutionalisation of Aboriginal Anthropology, Wentworth Conference. Canberra, AIATSIS.

RADCLIFFE-BROWN, Alfred R.

1951 « The Comparative Method in Social Anthropology », The Journal of the Royal Anthropological Institute of Great Britain and Ireland 81-1:15-22.

REAY, Marie, éd.

1964 Aborigines Now. New perspective in the study of Aboriginal Communities. Sydney, Angus and Robertson.

WHITLAM, Gough

1985 The Whitlam government, 1972-1975. Ringwood, Viking Penguin.

WOLFE, Patrick

1991 « On Being Woken Up : the Dreamtime in Anthropology and in Australian Settler Culture », Comparative studies in society and history $33: 197-224$

\section{NOTES}

1. Nous avons choisi de laisser une large place aux extraits de la lettre elle-même sans pour autant la reproduire entièrement.

2. Peter Thomson était marié à Lyn Thomson. Il avait fait des études d'archéologie au département d'anthropologie de l'Université de Sydney, sans poursuivre de carrière universitaire. La lettre souligne ainsi : «L'un d'entre nous était impliqué à titre professionnel dans les études aborigènes, jusqu'à ce que la contradiction entre la vision universitaire des Aborigènes et les expériences partagées in living avec des Aborigènes ne soit plus supportable. »

3. C'est dans le cadre d'un travail de thèse à l'école des Hautes études en Sciences Sociales mené sur le militantisme aborigène dans le sud-est de l'Australie à cette époque que nous avons pris connaissance de cette lettre. Nous avons pu interroger au cours de notre recherche Terry Widders, Gary Williams et Peter Thomson. Si la lettre n'était pas au cœur de notre recherche 
principale, elle a néanmoins été évoquée au cours des entretiens avec ces trois militants. Lyn Thomson est aujourd'hui décédée.

4. Le groupe a rédigé en avril 1975 un tract de deux pages pour dénoncer le plan « Homes for Aborigines ", qui consistait à isoler les familles aborigènes de Nouvelle-Galles du Sud (New South Wales, NSW) en leur proposant des maisons au milieu de familles «blanches ». C'est, à notre connaissance, la seule autre action clairement revendiquée par le groupe en tant que tel.

5. Voir Peter Tobin, « Aboriginal Land Rights in NSW », 1972 (in Goodall 1996).

6. Le caractère relativement éphémère du groupe n'en diminue pas l'importance dans le souvenir de ceux qui en furent membres. Il faut avoir conscience néanmoins que cette initiative s'inscrivait dans un ensemble plus large d'actions politiques.

7. En Australie, le «temps du rêve » et ses variantes ont aujourd'hui partie liée avec tout ce qui était ou reste aborigène. Pour plus de précision, voir notamment Barbara Glowczewski, 2004. Pour une discussion critique de la genèse du concept, voir Wolfe 1991. Pour une lecture de l'Eaglehawk and Crow par l'anthropologie sociale structurale, voir Radcliffe-Brown 1951.

8. Si cette "rareté" a partie liée avec l'insuffisance des sources, il nous semble que les orientations de recherche sont également en question. De nombreux autres documents de ce type sont probablement disponibles et leur étude pourrait être complétée par des enquêtes d'histoire orale.

9. Peter Thomson m'a ainsi expliqué que ce «partenariat entre des Aborigènes et des jeunes universitaires comme [lui]-même était très efficace [difficult to dismiss], parce que nous en savions suffisamment sur le pourquoi et le comment du fonctionnement de l'Institut et des études aborigènes ". Il serait, nous semble-t-il, également trompeur de réduire le contenu et l'initiative de la lettre à la seule influence de Thomson que d'ignorer complètement sa présence.

10. La conférence inaugurale de l'AIAS se tint à Canberra en 1961. L'annonce faite à la presse de la création de l'AIAS précisait ainsi : «Les occasions d'étudier la culture unique des aborigènes [sic] d'Australie diminuent rapidement. [...] Le Premier Ministre a souligné que la nouvelle institution prendrait en charge les aspects scientifiques, culturels et anthropologiques du problème. »

11. Richard Gould avait publié, malgré ses promesses de respecter la volonté de ses enquêtés, des images de cérémonies qui ne devaient pas être montrées à certaines personnes. Une lycéenne avait vu les photographies interdites, ce qui avait provoqué la colère des habitants.

12. Ce jeu de passage entre l'expression «les Aborigènes » et l'usage du «nous » au sein d'une même phrase est important, nous le verrons par la suite.

13. Le Ministre des Affaires aborigènes du gouvernement Gough Whitlam.

14. Cette stratégie de division manichéenne de l'espace social entre les «Blancs » d'un côté et les «Aborigènes » de l'autre est présente tout au long du texte. Les signataires insistent notamment sur le fait que les anthropologues sont forcément du côté des oppresseurs, et les Aborigènes forcément du côté des opprimés. Il est vrai que cette position était, dans le contexte de l'époque, plus facile à tenir : il n'y avait pas encore de classe moyenne aborigène.

15. Voir le tableau en annexe. Les réponses, reçues entre mars et mai 1974, ont été compilées par les membres du groupe, qui les ont fait circuler au moment de la conférence. S'il existe une copie de la lettre à la bibliothèque de l'AIAS, les réponses m'ont été fournies par Peter Thomson, qui en avait gardé un exemplaire.

16. L'ouvrage Aborigines Now. New Perspective in the Study of Aboriginal Communities (Reay 1964), regroupait les contributions d'une «nouvelle génération de chercheurs" dénonçant les penchants « antiquaires » de l'AIAS. On retiendra notamment les noms de Jeremy Beckett, Diane Barwick et Judy Inglis.

17. Gough Whitlam - dont l'élection marquait le retour des travaillistes au pouvoir après vingtcinq ans d'absence - déclarait ainsi lors de son investiture : "Non seulement aujourd'hui, mais dans la perspective plus large de l'Histoire, les Aborigènes imposent une responsabilité à laquelle 
nous ne pouvons pas échapper, que nous ne pouvons pas partager, dont nous ne pouvons pas nous débarrasser. Le monde ne nous laissera pas l'oublier.» (Whitlam 1985 : 466)

18. La "décolonisation interne » de l'Australie commença par l'octroi aux Aborigènes, tout au long des années 1960, des droits sociaux, économiques et politiques attachés à la condition de citoyen australien, qui leur étaient jusque-là refusés, aussi bien au niveau de l'état fédéral que des états fédérés. Par « ancien régime ", nous faisons référence au régime des Aboriginal Welfare Boards, sous la tutelle desquels vivaient les Aborigènes dans les différents États.

19. Voir par exemple « Friends of Blacks », The Adviser, 5 mai 1977, Adélaïde.

20. Si ces lettres sont minoritaires parmi celles reçues, on peut imaginer que la plupart des chercheurs hostiles à cette protestation n'ont pas pris le temps d'y répondre.

21. La réponse de Jim Cavanagh, ministre des Affaires aborigènes, reprenait ce dernier argument. 22. La comparaison est saisissante avec la discussion que relate Benoît de L'Estoile entre Marcel Griaule et un intellectuel africain, Taoré, à Genève en 1951. Alors que Taoré reprochait à Griaule de «parler des Noirs sans les rattacher à la réalité de leur histoire, de leur existence quotidienne ", expliquant qu'il « est plus urgent pour nous de réclamer du pain et la liberté pour les Africains que de renseigner les Européens sur le problème de la civilisation africaine», Griaule dénonçait en réponse « les Noirs évolués, qui parlent beaucoup pour ne rien dire et qui ne nous apprennent rien sur eux-mêmes sinon pour nous montrer leurs désirs » (De L'Estoile 1997).

23. La politique d'assimilation succédait à la politique de "protection", qui consistait principalement à parquer les Aborigènes dans des réserves en attendant qu'ils disparaissent. Voici comment l'Aboriginal Welfare Board la présente dans son rapport annuel pour 1952: «Une assimilation complète implique que l'Aborigène soit éduqué de façon à prendre sa place dans la structure sociale générale et s'adapte aux coutumes et aux principes de l'homme blanc. Par exemple, il doit acquérir la tolérance, et un mode de vie respectant les principes d'hygiène ordinaires et une connaissance de la valeur de l'argent. "

24. On notera que les communautés aborigènes du sud-est de l'Australie étaient très «métissées ». Ce métissage n'était pas tellement le fait d'union «mixtes » (Blanc/Aborigène), mais très largement la conséquence de mariages entre métis. De fait, quelle que soit leur couleur de peau, les membres de ces communautés se considéraient comme Aborigènes et étaient identifiés comme tels par la population blanche.

25. De même cette citation d'un fonctionnaire de l'administration aborigène chargé de la côte nord de la Nouvelle-Galles du Sud, d'où Gary Williams était originaire : «De façon générale, je trouve les Aborigènes trop absorbés par leurs propres problèmes pour s'intéresser à la vie sociale, culturelle ou civile de la communauté. Il est très rare d'en trouver un qui se détache du groupe et trouve un intérêt pour ce qui se passe en dehors de son propre cercle. Ceux qui jouent au football avec des équipes blanches retournent toujours trouver de la compagnie with their own people.» Dans la même veine, il est souvent fait référence à l'isolation complex [ « complexe d'isolement »] qui existerait à l'intérieur les communautés aborigènes.

26. «We grew up in the shadow of this." Gary Williams m'expliquait ainsi : "La dernière initiation majeure (avec des heavy duty men) a eu lieu en 1935. Le frère de ma grand-mère faisait partie de ces gens. Il faisait les initiations. »

27. Les analyses proposées par Richard Hoggard sur la classe ouvrière anglaise des années 1950 ou celles de Bernard Pudal sur les militants communistes de la période de bolchevisation du Parti communiste français permettent des comparaisons fécondes sur cette question du rapport autonomie/hétéronomie.

28. «L'espoir réside en l'éducation de la nouvelle génération », expliquait ainsi le Board en 1954. Il rappelait en 1962 que l'éducation était « un facteur décisif dans la mise en place de la politique d'assimilation ». [Ironie du sort,] dix ans plus tard, ce seront ces mêmes "enfants modèles ", 
devenus adolescents ou jeunes adultes, qui, s'inspirant du modèle noir américain, importeront en Australie le militantisme Black Power.

29. Il n'acheva jamais son premier cycle en droit. Len Watson venait, lui, du Queensland et était un peu plus âgé que les autres membres du groupe. Nous n'avons pas beaucoup d'informations biographiques à son sujet.

30. En 1951, le Board soulignait à la fois «l'absence d'intérêt des parents » et le « désir naturel des enfants eux-mêmes d'interrompre leur scolarité au plus vite». En 1962, cet organisme déplorait à nouveau la «tendance forte des boursiers aborigènes et des écoliers aborigènes d'arrêter l'école à peine atteint l'âge légal». En 1964, il rappelait que "l'absentéisme et le manque d'intérêt étaient un problème pour beaucoup d'élèves. Dans certains cas, les jeunes sont pressés de commencer à travailler, mais dans la majorité des cas, ils dérivent dans la vie communautaire facile, en vivant de travaux précaires et occasionnels ".

31. La population aborigène de Redfern passa de mille à plus de dix mille au cours des années 1960. La première initiative politique de ces militants fut la mise en place d'un groupe de surveillance de la répression policière (Pigs Patrol). Cette initiative donna lieu à la création d'un service d'aide juridique gratuit pour les Aborigènes et d'autres structures communautaires (aide médicale, garderie, etc.) furent mises en place par les Aborigènes eux-mêmes. Ces services servirent ensuite de modèle pour toute l'Australie.

32. L'épisode de l'ambassade-tente est leur principal fait d'armes. Le 26 janvier 1972 au matin, quatre militants aborigènes installèrent un parasol sur la pelouse devant le Parlement à Canberra, qu'ils appelèrent «l'ambassade aborigène ». La logique était la suivante: "Nous sommes traités comme des étrangers dans notre propre pays, nous avons donc droit à notre ambassade. » Cette dernière survécut six mois grâce à un vide juridique, puis fut démontée par la police à trois reprises, les 20,23 et 30 juillet. Il s'agissait néanmoins d'un coup politique extraordinaire, suscitant l'attention de la presse nationale et internationale.

33. La première action de ce type fut la prise de l'Aboriginal Advancement League dans le Victoria par Bruce McGuinness en janvier 1970.

34. Il faut souligner néanmoins le caractère limité - quantitativement et qualitativement - de leur intégration dans le champ politique. Dans de nombreux cas, des positions ad hoc étaient spécialement créées pour eux, et leur engagement était souvent confiné à des positions marginales de la bureaucratie et de la para-bureaucratie. Leur situation peut être résumée comme l'ascension exceptionnelle mais limitée d'un groupe minoritaire dans des positions marginales et précaires (voir notre travail de thèse en cours).

35. C'était une question relativement nouvelle dans la mesure où ce n'est qu'avec l'élection des travaillistes en décembre 1972 que le gouvernement du Commonwealth commença à assumer pleinement ses responsabilités et à injecter des sommes d'argent conséquentes dans les affaires aborigènes. La dénonciation par les militants d'une «industrie aborigène », dont les anthropologues feraient partie, est assez ancienne. Le militant Gary Foley aime ainsi à rappeler que « si, une nuit, tous les Aborigènes venaient à mourir, des milliers de Blancs se retrouveraient au chômage du jour au lendemain ».

36. L'idée même de l'Eaglehawk and Crow témoigne de leur goût pour la découverte, même si la motivation principale était clairement politique.

37. Les dialectes bandjalang étaient parlés dans la région où Gary Williams avait grandi. Les militants eux-mêmes n'avaient qu'une connaissance très réduite des langues, qui n'avaient pas été transmises au-delà de la génération de leurs grands-parents.

38. Roberts venait du Territoire du Nord. Il fut un des trois premiers Aborigènes engagés par le gouvernement australien comme agents de liaison.

39. Meredith Burgmann expliquait ainsi à Gary Foley, un des militants de l'époque : «à la fin de la campagne, j'avais vingt ou trente amis aborigènes, et je me sentais à l'aise et capable d'être impliquée dans les affaires aborigènes après 1971. J'avais toujours été sympathisante, mais je ne 
me sentais pas à l'aise, parce que je ne me sentais pas faire suffisamment partie de tout ça. Mais je pense que ça a aidé beaucoup d'étudiants blancs, parce qu'on se sentait plus... Tu sais, vous étiez nos amis, et donc on se sentait impliqué par ces questions... Ma vie sociale a aussi beaucoup changé. On allait à l'Empress Hotel, for Christ's sake, what a bloody help that was... "

40. "J'ai simplement décidé de ne pas devenir universitaire... Ce qui est toujours assez difficile, néanmoins... Il y a quelques années, je suis retourné faire quelques études d'archéologie... Et j'ai suivi ce cours théorique... Et il y avait tout un truc sur la critique postmoderne du monde universitaire... Et je me suis dit: "Si j'avais eu cette approche théorique ou analytique de la discipline quand j'étais impliqué en 73, j'aurais pu réussir à concilier certaines choses..." Mais je n'avais accès à aucune critique. Je n'avais pas d'autre choix que de partir... »

41. Il conviendrait de pousser plus avant l'enquête pour répondre précisément à cette question. Il faudrait notamment étudier plus en détail les débats non épistolaires qu'elle a suscités.

42. Nick Peterson, chercheur à l'Australian National University, est un des principaux anthropologues aboriginalistes australiens. La Wentworth Lecture est organisée par l'AIATSIS (l'AIAS devenu en 1989 The Australian Institute of Aboriginal and Torres Strait Islander Studies). Une autre anthropologue, Annette Hamilton, fait sensiblement le même constat. Cette nouvelle phase est également marquée par un recul de la domination de l'anthropologie sociale britannique sur l'anthropologie australienne du fait de l'arrivée de chercheurs américains.

43. En témoigne l'amertume de Bernard Moizo, dont la thèse fut interdite de publication. Pour lui, le contrôle local confine à la censure de l'anthropologie par les «bureaucraties ethniques »: «Les interventions d'organisations aborigènes via leurs représentants, le plus souvent non aborigènes, sont croissantes et ponctuent tous les stades de la recherche : élaboration d'un projet, obtention d'un permis, contrôle des relations anthropologue/informateurs, avis sur les travaux avant publication. Si l'aval d'une population pour une recherche qui les concerne et le retour de l'information se justifient pleinement, les interférences constantes, voire la censure exercée par certains au nom des Aborigènes sont tout aussi intolérables pour le chercheur que l'était le contrôle du gouvernement sur les travaux anthropologiques dans les années 1950.» (Moizo 1997)

44. On pourrait quasiment parler de changement de paradigme au sens où l'une des règles identifiées par Pierre Bourdieu comme faisant la spécificité du champ scientifique («le fait que les concurrents ne peuvent pas se contenter de se distinguer de leurs devanciers déjà reconnus, mais sont contraints, sous peine d'être dépassés et "déclassés", d'intégrer leurs acquis dans la construction distincte et distinctive qui les dépasse ») semblait ici suspendue.

45. La restitution de la complexité des relations nouées avec les interlocuteurs est aujourd'hui considérée comme une composante indispensable des analyses produites par l'ethnographe.

46. Berndt 1982. Ronald Berndt était un des grands anthropologues aboriginalistes australiens et avait commencé sa carrière dans les années 1940. Un des problèmes que posent ce genre de textes, écrits par des anthropologues sur l'histoire de leur discipline, est qu'ils entrecroisent formes rituelles de la mémoire et formes savantes de l'histoire. Benoît de L'Estoile suggère ainsi un parallèle entre histoire disciplinaire et histoire locale en tant que modalité d'appropriation du passé.

47. De la même manière, le rôle actif et l'influence des colonisés sur les colonisateurs et sur la situation coloniale ont longtemps été occultés. Des historiens comme Frederick Cooper ont pourtant montré que les "policy and ideology also reflected European adaptation (and resistance) to the initiative of the colonised».

48. Cette porosité qui, dans certains contextes (crises politiques, refondation d'un régime, alternances politiques), caractérise le discours savant de l'anthropologie ne concerne pas uniquement les résistances anticoloniales. La thèse de Benoît de L'Estoile dévoile ainsi des processus de traduction d'intérêts définis comme "pratiques» en questions reconnues comme « scientifiques ", à partir du cas de l'anthropologie malinowskienne. 
49. Les deux discours suscitaient probablement chez lui une égale aversion.

50. On pourrait même faire l'hypothèse que ce type de critique ne pouvait être le fait que d'individus ne partageant pas les caractéristiques sociales des anthropologues de l'époque. Les socialisations « contradictoires » communes aux membres du groupe leur donnaient, semble-t-il, une position privilégiée pour mettre en cause, de façon certes plus pratique que théorique, le discours anthropologique. Ann Laura Stoler et Frederick Cooper, reprenant un thème de Paul Gilroy, expliquent que : "Slavery, colonialism and racism gave those who experienced them a vantage point on modernity that starkly revealed the limits of the economic progress, political participation and social inclusiveness. "

51. La stratégie des prétendants, à l'intérieur du monde anthropologique, relevait, semble-t-il, autant de la subversion que de la succession. La redéfinition de l'ordre scientifique établi permettait de marginaliser la «vieille garde " tandis que le maintien des institutions permettait de se prémunir contre une remise en cause trop forte des principes de légitimation de la domination et par là même contre une incursion d'outsiders.

52. Il n'est pas interdit de penser que l'autonomie du champ scientifique vis-à-vis du champ militant était beaucoup plus grande que la réciproque. Le discours militant, souvent amené à faire feu de tout bois, est en effet moins protégé que le discours savant. Il conviendrait ainsi d'interroger en détail les effets en retour de l'anthropologie sur le mouvement aborigène et notamment sur la structuration des revendications politiques. Les modèles de revendication (Black Power aux États-Unis, mouvements de libération et de décolonisation en Afrique et en Asie, etc.) utilisés par les militants aborigènes étaient dans un premier temps assez éloignés de la mise en avant de la seule « autochtonie ».

53. Nous l'avons vu, les spécificités du contexte sont à observer à la fois à l'échelle des structures - la modification des rapports de force à l'intérieur de l'ordre politique vis-à-vis duquel le champ scientifique était marqué, dans le cas de l'anthropologie, par une dépendance certaine - mais aussi dans la particularité des trajectoires des acteurs et de leurs positions à l'intérieur des champs scientifique et politique.

\section{RÉSUMÉS}

En mars 1974, un groupe de militants aborigènes intitulé "The Eaglehawk and Crow », envoya une lettre de protestation aux chercheurs participant à l'assemblée de l'Institut Australien des Études Aborigènes. Cette lettre, qui constituait une mise en question radicale des pratiques anthropologiques de l'époque, provoqua de nombreuses réactions de la part des personnes mises en cause.

Notre article se propose, à partir d'une analyse du document lui-même mais aussi des réponses qu'il a suscitées, de réfléchir à l'impact de protestations extérieures telles que celle-ci sur l'évolution des études aborigènes. L'anthropologie, comme science, n'a pas uniquement évolué à la suite de débats entre anthropologues, mais également $d u$ fait d'oppositions venant d'agents extérieurs à la discipline, et notamment de certaines fractions des populations colonisées.

«An open letter concerning the Australian Institute of Aboriginal Studies» signed by the Eaglehawk and Crow was sent to the March 1974 meeting of the AIAS. This letter questioned the basic anthropological practices of the time, provoking considerable reaction from those addressed. This article is based on an analysis of the letter itself and on the responses it aroused 
and considers the impact of protests from those outside the science such as this letter about the development of aboriginal studies. Anthropology, like science, has not evolved solely as a result of discussion between anthropologists but also in response to reactions from those outside the discipline, in particular certain groups within colonised peoples.

INDEX

Keywords : Aborigines, anthropology, Australia, militancy, politics, science

Mots-clés : Aborigènes, anthropologie, Australie, militantisme, politique, science

\section{AUTEUR}

\section{BASTIEN BOSA}

Genèse et transformation des mondes sociaux (GTMS), EHESS, Laboratoire de Sciences Sociales, ENS, bastien.bosa@ens.fr 